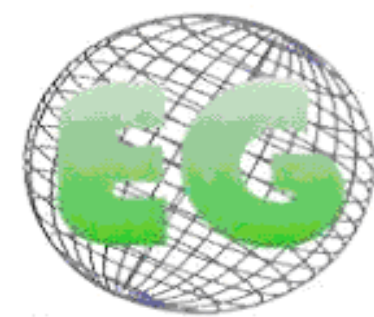

ISSN 1695-6141 $N^{\circ} 22$
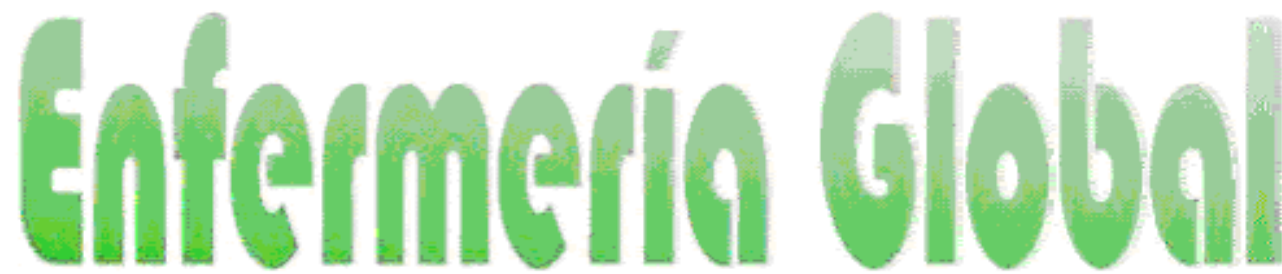

Revista electrónica trimestral de Enfermería

Abril 2011

www.um.es/egloball

\title{
DIAGNÓSTICOS ENFERMEROS EN UFISS, UGA, TRAUMATOLOGÍA Y CIR.
}

NURSING DIAGNOSES IN SOCIAL-SANITARY FUNTIONAL INTERDISCIPLINARY, GERIATRICS , TRAUMATOLOGY AND GENERAL SURGERY.

\author{
*Luque Ballesteros,M., Micó Reyes, E., Tantiñá Fontanet, S., Moreiras Andrino, \\ MC., Díaz García, L., Sabater Raga, R.
}

\begin{abstract}
*Enfermeras de la Fundación Hospital Asilo Granollers. Barcelona.
Palabras clave: Diagnósticos enfermeros; Taxonomía NANDA; Implantación informática. Keywords Nursing diagnoses; Taxonomia NANDA; computer implementation
\end{abstract}

\section{RESUMEN}

Introducción: La utilización de un método validado, ha permitido detectar las necesidades alteradas en relación a su etiología, definiendo los DdE (Diagnósticos de Enfermería) (3).

La utilización de la Taxonomía NANDA 2 asegura la definición de la respuesta humana a un problema tanto dentro del marco profesional como jurídico, así mismo permite un lenguaje común en la práctica enfermera (6).

Objetivos. Identificar los DdE más prevalentes en la población atendida por la UFISS (Unidad Funcional Interdisciplinar Socio-Sanitaria), UGA (Unidad de Geriatría Aguda), TRAUMATOLOGÍA y CIRUGÍA de la FHAG (Fundación Hospital Asilo de Granollers) utilizando la taxonomía NANDA.

\section{Métodos.}

UFFIS. Se estudian retrospectivamente todas las historias de la UFISS del año 2006. (674 consultas, entrando en estudio $\mathrm{N}=390$ estudiadas).

Se estudian prospectivamente:

COT (Cirugía Ortopédica Traumatológica). 24 pacientes geriátricos de la unidad de trauma ingresados durante los meses de Agosto-Septiembre 2008

UGA. 49 pacientes ingresados durante los meses de Septiembre-Diciembre 2008

CIR. 36 pacientes ingresados en mayo 2009.

Valoración paciente: Abordaje Bio-Psico-Social (Entrevista enfermera al paciente y al cuidador principal).Funcional (Barthel). Instrumentales (Lawton). Cognitivo (Pfeiffer). Riesgo de úlceras (EMINA). Dolor (EVA)

\section{Resultados.}

UFISS: Se detectan 18 diagnósticos, como los más prevalentes valorados en la UFISS, 
COT: Se detectan 26 diagnósticos, 16 son comunes a los recogidos por la enfermera de la UFISS, los 10 restantes son los específicos detectados en el paciente orto geriátrico:

UGA: Se detectan 30 diagnósticos, de los cuales 18 son comunes a la UFISS y los 12 restantes son específicos en el paciente geriátrico.

CIR: Se detectan alrededor de 50 diagnósticos; pendiente tabulación final.

Conclusiones: Se han definido los diagnósticos más prevalentes determinando los comunes a las diferentes áreas asistenciales. Dado que en nuestra institución la formación es mayoritariamente básica, con este estudio hemos conseguido: 1) difundir el lenguaje NANDA, 2) asegurar el dominio de estos diagnósticos, 3) que el profesional trabaje de forma más segura utilizando un lenguaje validado y entendible y 4) orientar a la futura implantación informática.

\section{ABSTRACT}

In 2006, after the addition of a new nurse in UFISS (Social-Sanitary Functional Interdisciplinary Unit), is detected the need of a common language for all nurses with which to conduct a data collection for the nursing reports. The aim of this study is to know the main nursing diagnoses in UFISS, Geriatrics, Traumatology and Surgery units, using the NANDA (North American Nursing Diagnosis Association) nursing diagnostic terminology. We performed a retrospective study of all the medical reports at UFISS in 2006, a prospective study of all medical reports at Geriatrics and geriatric patients at Traumatology and Surgery in various periods between 2008 and 2009. According to the results, the use of a validated method like NANDA diagnoses, has enabled to nurses to identify common altered needs and after that, to define Nursing Diagnoses and develop the optimal care plan for the patient

\section{INTRODUCCIÓN}

El diagnóstico de enfermería es un juicio clínico de una valoración ${ }^{(1)}$. "Es un juicio clínico sobre la respuesta de una persona, familia o comunidad a etapas de la vida / problemas de salud reales o potenciales, que el enfermero identifica, valida y trata de forma Independiente" (AENTDE, mayo de 2001).

La principal organización en el desarrollo enfermero es la North American Nursing Diagnosis Association (NANDA internacional), en España es la Asociación Española de Nomenclatura, Taxonomía y Diagnóstico de Enfermería (AENTDE) ${ }^{(2)}$.

El diagnóstico es un juicio clínico de valoración de un problema real o potencial que las enfermeras están autorizadas y son legalmente responsables para $\operatorname{tratar}^{(3)}$.

La valoración ha sido realizada por una enfermera de la UFISS, que tiene las habilidades suficientes para ver al paciente como ser integral (bio-psico-social). Es un proceso ordenado y sistemático de recogida de datos, registro y elaboración de los diagnósticos ${ }^{(4)}$. Los datos se recogen de la historia médica, de la exploración física, de la valoración de otros profesionales y de la entrevista paciente/familia/cuidador ${ }^{(5)}$.

El sistema de clasificación diagnóstica es el de la NANDA ${ }^{(6)}$, con el que se formula en dos partes: problema y etiología, ya que para poder actuar tenemos que saber la causa.

\section{OBJETIVOS}

1. Identificar los Diagnósticos enfermeros más prevalentes en la población atendida por la UFISS, GERIATRÍA, TRAUMATOLOGÍA Y CIRUGÍA FHAG utilizando la taxonomía NANDA. 
2. Crear un dossier de los diagnósticos más prevalentes.

3. Realización de sesiones informativas.

4. Facilitar a la institución la información necesaria para crear el aplicativo informático de enfermería.

\section{MATERIAL Y MÉTODO:}

- Se estudian retrospectivamente todas las historias de la UFISS del año 2006. (674 consultas, 284 excluidas por tratarse de intervenciones médicas. $\mathrm{N}=390$ estudiadas).

- Se estudian prospectivamente todos los pacientes geriátricos de la unidad de trauma ingresados durante los meses de Agosto-Septiembre 2008 (24 pacientes con criterio de valoración de UFISS. $\mathrm{N}=24$ )

- Se estudian prospectivamente todos los pacientes de la unidad de geriatría de agudos durante los meses de Septiembre-Diciembre 2008 ( $\mathrm{N}=49$ pacientes).

- Se estudian prospectivamente todos los pacientes de la unidad de cirugía durante el mes de Diciembre 2009 ( $\mathrm{N}=36$ pacientes).

Valoración paciente:

Abordaje Bio-Psico-Social (Entrevista enfermera al paciente y al cuidador principal).

Funcional (Barthel)

Instrumentales (Lawton)

Cognitivo (Pfeiffer)

Riesgo de úlceras (EMINA)

Dolor (EVA)

\section{RESULTADOS}

\section{Resultados UFISS}

Se detectan 18 diagnósticos, como los más prevalentes valorados en la UFISS,.

> Baja autoestima situacional 00120

> Riesgo del síndrome del desuso 00040

> Trastorno de la imagen personal 00118

> Trastorno de la percepción sensorial 00122

> Deterioro de la interacción social 00052

$>$ Deterioro de la comunicación verbal 00051

> Déficit auto cuidado: vestido/acicalamiento 00109

> Deterioro del patrón del sueño 00095

> Afrontamiento familiar inefectivo 00069

> Riesgo del cansancio en el rol del cuidador 00062

> Déficit de la movilidad física 00085

> Déficit de auto cuidado: alimentación 00102

$>$ Deterioro de la deglución 00103

> Patrón respiratorio ineficaz 00032

$>$ Incontinencia urinaria total 00021

$>$ Estreñimiento 00011

> Riesgo de deterioro de la integridad cutánea 00047

> Alteración de procesos mentales: confusión aguda 00128

confusión crónica 00129 
Siete de los cuales se dan en más de un $50 \%$ de los pacientes:

- Riesgo deterioro integridad cutánea 97,

- Estreñimiento $94,9 \%$

- Déficit movilidad $94,1 \%$

- Riesgo Síndrome del desuso $77,7 \%$

- Afrontamiento familiar ineficaz $61,5 \%$

- Alteración procesos mentales $53,8 \%$

- Baja autoestima situacional 53,6

\section{Resultados COT}

Para valorar los diagnósticos enfermeros detectados por una enfermera asistencial, se realiza un estudio prospectivo durante Agosto y Septiembre del 2008 en la unidad de traumatología, son 24 los pacientes geriátricos ingresados en la unidad siendo esta la que hace la valoración.

Se detectan 26 diagnósticos, 16 son comunes a los recogidos por la enfermera de la UFISS, los 10 restantes son los específicos detectados en el paciente ortogeriátrico:

Ansiedad 00146

> Riesgo de caídas 00155

$>$ Conocimientos deficientes 00126

$>$ Diarrea 00013

$>$ Dolor agudo 00132 (+ 3 en escala EVA)

$>$ Dolor crónico 00133

$>$ Hipertermia 00006

$>$ Riesgo de infección 00004

$>$ Intolerancia a la actividad 00092

$>$ Riesgo de desequilibrio de volumen de líquidos 00025

\section{Resultados Geriatría}

Para valorar los diagnósticos enfermeros detectados por una enfermera asistencial, se realiza un estudio prospectivo durante Septiembre y Diciembre del 2008 en la unidad de geriatría de agudos, se escogen los pacientes geriátricos y es esta la que hace la valoración. Entran dentro del estudio 49 pacientes por criterio de inclusión.

Se detectan 30 diagnósticos, 18 son comunes a la UFISS y los 12 restantes son los específicos en el paciente geriátrico:

\section{Fatiga 00093}

Déficit auto cuidado: uso wc 00110

> Deterioro de la movilidad en la cama 00091

> Deterioro de la movilidad en silla de ruedas 00089

$>$ Deterioro en habilidad translación 00090

> Desequilibrio nutricional por defecto 00002

$>$ Desequilibrio nutricional por exceso 00001

$>$ Estreñimiento 00011

> Sufrimiento espiritual 00066

$>$ Riesgo de lesiones 00035

> Riesgo de caídas 00115 


\section{Resultados Cirugía}

Para valorar los diagnósticos enfermeros detectados por dos enfermeras asistenciales de la unidad de cirugía mayor, se realiza un estudio prospectivo estudiando todos los pacientes ingresados en esta, durante el mes de Diciembre. Entran dentro del estudio 36 pacientes.

Se detectan 26 diagnósticos:

Riesgo de lesiones 00035

$>$ Riesgo de caídas 00155

$>$ Conducta generadora de salud 00084

$>$ Deterioro de .la integridad cutánea 00047

$>$ Riesgo de desequilibrio de volumen de líquidos 00025

$>$ Hipertermia 00007

> Deterioro de la deglución 00103

> Desequilibrio nutricional por defecto 00002

> Estreñimiento 00011

> Riesgo de estreñimiento. 00015

$>$ Diarrea 00013

$>$ Incontinencia urinaria 00021

$>$ Retención urinaria.00023

> Limpieza ineficaz de las vías aéreas

$>$ Riesgo de aspiración.00039

> Patrón respiratorio ineficaz.00032

> Déficit de la movilidad física 00085

> Deterioro de la movilidad en la cama 00091

> Deterioro de la deambulación. 00088

> Déficit de autocura: uso del WC 00110

$>$ Déficit autocura: Baño-higiene. 00108

$>$ Insomnio 00095

$>$ Dolor agudo 00132

> Trastorno de la imagen corporal 00118

$>$ Ansiedad 00146

$>$ Disfunción sexual. 00059

4 de los cuales se dan en más de un $50 \%$ de los pacientes:

Dolor agudo $100 \%$

Hipertermia $72,22 \%$

Deterioro integridad cutánea $69,44 \%$

$>$ Riesgo de lesiones $63,89 \%$

En este estudio se han detectado nuevos diagnósticos, y esto es debido a las características de los pacientes de esta unidad. Llama la atención que el DdE Disfunción Sexual se detecta únicamente en este servicio en un $8,33 \%$. 


\section{COMPARATIVA DIAGNÓSTICOS}

G

\begin{tabular}{|c|c|c|c|c|c|}
\hline PATRONS & DIAGNOSTICS INFERMERS & GERIATRIA & ORTOGERIATRIA & UFISS & CIRURGIA \\
\hline \multirow{3}{*}{$\begin{array}{l}\text { 1. Percepció de } \\
\text { la salut. }\end{array}$} & Risc de lesions 00035 & $\mathbf{x}$ & & & $\mathbf{x}$ \\
\hline & Risc de caigudes 00155 & $\mathbf{x}$ & $\mathbf{x}$ & $\mathbf{x}$ & $\mathbf{x}$ \\
\hline & Risc d'aspiració 00039 & & & & $\mathbf{x}$ \\
\hline \multirow{6}{*}{$\begin{array}{l}\text { 2. Nutricional } \\
\text { Metabòlic }\end{array}$} & Deteriorament integritat cutània 00047 & $\mathbf{x}$ & $\mathbf{x}$ & $\mathbf{x}$ & $\mathbf{x}$ \\
\hline & Risc de desequilibri de volum de líquids 00025 & & $\mathbf{x}$ & & $\mathbf{x}$ \\
\hline & Hipertermia 0006 & & $\mathbf{x}$ & & $\mathbf{x}$ \\
\hline & Deteriorament de la deglució 00103 & $\mathbf{x}$ & $\mathbf{x}$ & $\mathbf{x}$ & $\mathbf{x}$ \\
\hline & Desequilibri nutricional per defecte 00002 & $\mathrm{x}$ & & & $\mathbf{x}$ \\
\hline & Desequilibri nutricional per excès 00001 & $\mathbf{x}$ & & & \\
\hline \multirow{5}{*}{ 3.Eliminació } & Restrenyiment 00011 & $\mathbf{x}$ & $\mathbf{x}$ & $\mathbf{x}$ & $\mathbf{x}$ \\
\hline & Risc de restrenyiment 00015 & & & & $\mathbf{x}$ \\
\hline & Incontinència urinaria 00021 & $\mathrm{x}$ & $\mathbf{x}$ & $\mathbf{x}$ & $\mathbf{x}$ \\
\hline & Retenció urinària 00023 & & & & $\mathbf{x}$ \\
\hline & Diarrea 00013 & & $\mathbf{x}$ & & $\mathbf{x}$ \\
\hline \multirow{14}{*}{$\begin{array}{l}\text { 4. Activitat } \\
\text { Excercici }\end{array}$} & Neteja inefectiva de les vies aèries 00006 & $\mathbf{x}$ & $\mathbf{x}$ & $\mathbf{x}$ & $\mathbf{x}$ \\
\hline & Patró respiratori ineficaz 00032 & & & & $\mathbf{x}$ \\
\hline & Dèficit de la mobilitat física 00085 & $\mathbf{x}$ & $\mathbf{x}$ & $\mathbf{x}$ & $\mathbf{x}$ \\
\hline & Deterior de la deambulació 00088 & & & & $\mathbf{x}$ \\
\hline & Dèficit d'autocura alimentació 00102 & $\mathbf{x}$ & $\mathbf{x}$ & $\mathbf{x}$ & \\
\hline & Dèficit d'autocura en vestir-se 00109 & $\mathbf{x}$ & $\mathbf{x}$ & $\mathbf{x}$ & \\
\hline & Fatiga 00093 & $\mathrm{x}$ & & & \\
\hline & Dèficit d' autocura: us de WC 00110 & $\mathbf{x}$ & & & $\mathbf{x}$ \\
\hline & Dèficit d'autocura: bany / higiene 00108 & & & & $\mathbf{x}$ \\
\hline & Deterior de la mobilitat en el llit 00091 & $\mathbf{x}$ & & & $\mathbf{x}$ \\
\hline & Deterior en la mobilitat en cadira de rodes 00089 & $\mathbf{x}$ & & & \\
\hline & Risc de la sindrome del desuso 00040 & $\mathbf{x}$ & $\mathbf{x}$ & $\mathbf{x}$ & \\
\hline & Deterior en la habilitat per la traslació 00090 & $\mathbf{x}$ & & & \\
\hline & Intolerància a l'activitat 00092 & & $\mathbf{x}$ & & \\
\hline \multirow{2}{*}{ 5. Son Descans } & Insomni 00095 & & & & $\mathbf{x}$ \\
\hline & Alteració patró de la son 00095 & $\mathbf{x}$ & $\mathbf{x}$ & $\mathbf{x}$ & \\
\hline \multirow{6}{*}{$\begin{array}{l}\text { 6.Cognitiu } \\
\text { perceptiu }\end{array}$} & Confusió aguda 00128 & $\mathbf{x}$ & $\mathbf{x}$ & $\mathbf{x}$ & \\
\hline & Confusió crónica 00129 & $\mathbf{x}$ & $\mathbf{x}$ & $\mathbf{x}$ & \\
\hline & Transtorn de la percepció sensorial 00122 & $\mathbf{x}$ & $\mathbf{x}$ & $\mathbf{x}$ & \\
\hline & Dolor agut 00132 & & $\mathbf{x}$ & & $\mathbf{x}$ \\
\hline & Coneixements deficients 00126 & & $\mathbf{x}$ & & \\
\hline & Dolor crónic 00133 & & $\mathbf{x}$ & & \\
\hline \multirow{3}{*}{$\begin{array}{l}\text { 7.Autopercepció } \\
\text { Autoconcepte }\end{array}$} & Trastorn de la imatge personal 00118 & $\mathbf{x}$ & $\mathbf{x}$ & $\mathbf{x}$ & $\mathbf{x}$ \\
\hline & Baixa autoestima situacional 00120 & $\mathbf{x}$ & $\mathbf{x}$ & $\mathbf{x}$ & \\
\hline & Ansietat 00146 & & $\mathbf{x}$ & & $\mathbf{x}$ \\
\hline \multirow{3}{*}{ 8. Rol i relacions } & Sobre esforç en el rol de cuidador 00062 & $\mathbf{x}$ & $\mathbf{x}$ & $\mathbf{x}$ & \\
\hline & Dèficit de comunicació verbal 00051 & $\mathbf{x}$ & $\mathbf{x}$ & $\mathbf{x}$ & \\
\hline & Deteriorament de la interacció social 00052 & $\mathbf{x}$ & $\mathbf{x}$ & $\mathbf{x}$ & \\
\hline $\begin{array}{l}\text { 9. Sexualitat i } \\
\text { reproducció }\end{array}$ & Disfunció sexual & & & & $\mathbf{x}$ \\
\hline \multirow[t]{2}{*}{ 10. Tolerància } & Afrontament familiar ineficaç 00069 & $\mathbf{x}$ & $\mathbf{x}$ & $\mathbf{x}$ & \\
\hline & Sindrome $d^{\prime}$ estress per trasllat 00114 & $\mathbf{x}$ & & $\mathbf{x}$ & \\
\hline $\begin{array}{l}\text { 11. Valors i } \\
\text { creences }\end{array}$ & Patiment espiritual 00066 & $\mathbf{x}$ & & & \\
\hline
\end{tabular}




\section{CONCLUSIONES}

La utilización de un método validado, ha permitido a enfermería detectar las necesidades alteradas en relación a su etiología, definiendo los Diagnósticos Enfermeros. (Las enfermeras están autorizadas y son legalmente responsables para tratarlos)

El trabajo de la enfermera asistencial y la de UFISS, es un trabajo en equipo en el que cada uno de los profesionales detecta diferentes diagnósticos enfermeros y cada profesional interviene en cada uno de ellos consensuado y elaborando el plan de cuidados optimo para el paciente.

Debido a que la gran mayoría del personal de enfermería de la FHAG,no ha tenido en su plan de estudios la formación necesaria sobre los diagnósticos enfermeros, creemos necesario ampliar este trabajo al resto de las unidades del centro, para asegurarnos el dominio correcto de los diagnósticos más prevalentes de toda la FHAG.

\section{BIBLIOGRAFÍA}

1.- Iyer PW, Tapich BJ, Bernocchi-Losey D. Proceso de Enfermería y diagnostico de Enfermería. Madrid: Interamericana. McGraw-Hill, 1998.

2.- Johnson, Bulechek, Butcher, McClosekey Dochterman, Maas, Moorhead, Swanson. Interrelaciones NANDA, NOC y NIC. Madrid: Eselvier España 2007.

3.- Cuesta Zambrana A, Guirao Goris JF, Benavent Garcea A. Diagnósticos de Enfermería. Madrid: Diaz Santos 1994.

4.- Gil VF, Merino J, Orozco D. Manual de metodología de trabajo en enfermería. En Almansa Martínez, profesora titular fundamentos de enfermería, Universidad Murcia. Madrid: Merck Sarph \& Dome, 1997. pXXIV/362-5.

5.- Gil VF, Merino J, Orozco D. Manual de metodología de trabajo en enfermería. En Pérez Garcia RB. Diplomada Universitaria en Enfermería. Madrid: Merck Sarph \& Dome, 1997. pXXIV/225-8.

6.- Ugalde Apalategui M, Rigol i Cuadra A. Diagnósticos de Enfermería Taxonomía NANDA. Barcelona: Masson SA, 1995. 